\title{
Analysis of R-band Observations of an Outburst of Comet 29P/Schwassmann-Wachmann 1 to Place Constraints on the Nucleus' Rotation State
}

\author{
Charles A. Schambeau $^{\mathrm{a}}$, Yanga R. Fernández ${ }_{1}^{\mathrm{a}}{ }$, Nalin H. Samarasinha ${ }^{\mathrm{b}}$, Beatrice E.A. Mueller ${ }_{1}{ }^{\mathrm{b}}$, Laura M. Woodney ${ }^{\mathrm{c}}$ \\ ${ }^{a}$ Department of Physics, University of Central Florida, Orlando, FL 32816, USA \\ ${ }^{b}$ Planetary Science Institute, Tucson, AZ 85719, USA \\ ${ }^{c}$ Department of Physics, California State University San Bernardino, San Bernardino, CA 92407
}

\begin{abstract}
We present analysis of five nights of R-band observations of Comet 29P/Schwassmann-Wachmann 1 (SW1) taken on September 2008 which show the comet undergoing an outburst. Coma morphology shows a projected asymmetric shell of material expanding radially and four linear features on the northern side of the coma at position angles $37^{\circ}, 78^{\circ}, 300^{\circ}$, and $353^{\circ}$. Using the measured projected radial outflow velocity of $0.11 \pm 0.02 \mathrm{~km} / \mathrm{s}$ for the shell material, we calculate an outburst time of UT 2008-09-21.03 \pm 0.95 days. By tracking the inner and outer extent of the northern linear features, we found that the features are fully contained within the expanding shell of material. This suggested both shell and linear features originated during the same event and activity originating from different regions on the nuclear surface are not necessary to generate both types of morphological structure observed. A 3-D Monte Carlo coma model was used to model the outburst. Morphological features present in the observations were modeled allowing constraints to be placed on the spin state of SW1's nucleus. The evolution of morphological features allows constraints on the rotation period $\mathrm{P}$ assuming an outburst duration $\Delta t$ and the spin period constraints are expressed in terms of their ratio $\mathrm{P} / \Delta t$. Since the spin-pole orientation could not be constrained, four spin-pole orientations were chosen for modeling the coma. Spin-period constraints for each assumed pole orientation are discussed. Overall, modeling suggested either a spin period on the order of days, a spin-pole orientation nearly along the sub-Earth direction, or a combination of both. To place an independent constraint on the outburst duration, radial surface-brightness profiles of the observations were compared with profiles from synthetic models, giving an upper-limit of $\Delta t \leq 1.5$ days. Longer outbursts resulted in a higher number of dust grains in close proximity to the nucleus during the observations and a profile slope too steep to model observations. Lastly, from photometry of the five nights of observation, a lower limit of $(1.8 \pm 0.07) \times 10^{9} \mathrm{~kg}$ was estimated for the total amount of dust emitted during the outburst. Assuming the outburst was triggered by either the sublimation of pure $\mathrm{CO}_{\text {or }} \mathrm{CO}_{2}$ ice and a dust to gas ratio of $\sim 4$ (Rosetta results for Comet 67P, Rotundi et al. 2015), a lower limit for the outburst duration on the order of hours was obtained.
\end{abstract}

Keywords:

Comets: general, Comets: individual (29P/Schwassmann-Wachmann 1), Optical Observations

\section{Introduction}

Comet 29P/Schwassmann-Wachmann 1 (SW1) is an excellent observational target for understanding cometary nuclei and their activity drivers. SW1 is in a nearly circular orbit ( heliocentric distance ranges from 5.76-6.26 AU) with the following orbital parameters: eccentricity $=0.041830$, semi-major axis $a=6.009674 \mathrm{AU}$, and inclination $i=9.3763^{\circ}$ 
(IAU Minor Planet Center, Epoch 2014 Dec. 9.0 TT). The orbital properties of SW1 also classify it as a Centaur using the definition from Jewitt 2009. With a continuous background coma believed to be driven by the supervolatile CO (Festou et al. 2001; Gunnarsson et al. 2001; Paganini et al. 2013) and/or from gases released during the crystallization of amorphous water ice (Jewitt 2009), SW1 undergoes frequent outbursts in activity with increases in brightness ranging over several orders of magnitude and lasting from several days to a few weeks (Trigo-Rodriguez et al. 2010; Kossacki and Szutowicz 2013; Hosek et al. 2013; Miles et al. 2015). SW1's nearly circular orbit and thus an environment with nearly constant insolation raises the question of which activity drivers are causing the outbursts. With a seemingly stable thermal environment why would the nucleus experience frequent excursions from a steady state of activity? This puzzling behavior marks SW1 as an excellent target for continued observations and analysis.

Determining the spin state of an active comet nucleus is often a daunting task. With observations of the bare nucleus obstructed by the coma, direct links between the photometric light curve dominated by the coma and nucleus spin state become challenging. Variability in activity levels can mimic magnitude fluctuations caused by the geometric cross-section modulation during the rotation of an aspherical nucleus. Another method useful for placing constraints on the spin state of an active comet nucleus is to track changes in the coma morphology during a series of observations (Sekanina and Larson 1984; Samarasinha 2000; Schleicher and Woodney 2003; Farnham 2009; Mueller et al. 2013). Features in the coma (i.e. jets and shells of material) and their evolution can indicate the spin-pole orientation and/or rotation period. By tracking such features and their evolution during a time series of observations, inferences on the spin state can be made and used as initial estimates for input parameters in 3-D coma modeling routines.

By observing the evolution of SW1's coma during an outburst in a set of R-band observations from 2008 we place constraints on the time of the outburst, the 3-D shape of the outflowing outburst material, the nuclear spin state, the location of surface areas of activity involved in the outburst, and the duration of the outburst. Section 2 gives an overview of the observations, Section 3 describes the image enhancement techniques applied to the images and results from the coma morphology analysis, Section 4 is a description of the Monte Carlo 3-D coma simulation code along with its application to SW1, and finally Section 5 discusses estimates of the amount of dust emitted during the outburst. The paper is concluded with a discussion of the spin state of SW1 and its implications for future thermophysical modeling of the enigmatic comet SW1.

\section{Observations}

R-band observations were acquired on 2008 September 25-29 UT from the Kitt Peak 2.1-m telescope while SW1 was undergoing an outburst. The $2048 \times 2048$ T2KB CCD was used for the observations. Table 1 gives details about each night of observations and Figure 1 shows a sample image from each night. Standard image processing including bias subtraction, flat fielding, and background subtraction were applied to each, generating science quality images for the analysis. Absolute flux density calibration was performed on the observations using data products from the Sloan Digital Sky Survey (SDDS). The orientation of the images in this paper is equatorial north up and east to the left with a pixel scale of $0.3 \mathrm{arcsec} /$ pixel $(\sim 1400 \mathrm{~km} /$ pixel projected sky-plane distance at the location of SW1). The individual $240 \mathrm{~s}$ image frames were used for analysis and no co-addition of frames from each night was applied.

As can be seen in the enhanced images (Figure 2), a projected shell of material is radially outflowing due to the outburst. In addition to the shell of material, there are four linear features on the northern side of the coma at position 


\section{SW1 Observation Summary for UT September 2008}

\begin{tabular}{lccccc}
\hline \hline UT day & From & To & {$\left[\mathrm{R}_{h}, \Delta\right]^{\mathrm{a}}$} & Expo. Time $^{\mathrm{b}}$ & \# of Expo. $^{\mathrm{c}}$ \\
& $(\mathrm{UT})$ & $(\mathrm{UT})$ & $(\mathrm{AU})$ & $(\mathrm{s})$ & \\
\hline 25 & $11: 18: 23$ & $11: 38: 56$ & {$[6.064,6.437]$} & 240 & 3 \\
26 & $11: 31: 01$ & $11: 49: 53$ & {$[6.065,6.423]$} & 240 & 3 \\
27 & $11: 08: 44$ & $12: 15: 31$ & {$[6.065,6.408]$} & 240 & 8 \\
28 & $11: 47: 29$ & $12: 08: 00$ & {$[6.065,6.394]$} & 240 & 3 \\
29 & $11: 41: 29$ & $11: 59: 45$ & {$[6.065,6.379]$} & 240 & 3 \\
\hline
\end{tabular}

a Average heliocentric and geocentric distance during each night of observations (Horizons, JPL).

b Exposure time for each image frame.

${ }^{c}$ Number of SW1 images for the given night of observations.

angles (PA) $37^{\circ}, 78^{\circ}, 300^{\circ}$, and $353^{\circ}$ measured from north through east (Figures 3 and 4). Morphological features such as these, which allow characterization of the outburst and measurement of properties of the underlying nucleus, are the focus of this paper. As the coma features are subtle, a suite of image enhancement routines were used on the images, bringing out details helpful for the characterization of the outburst.

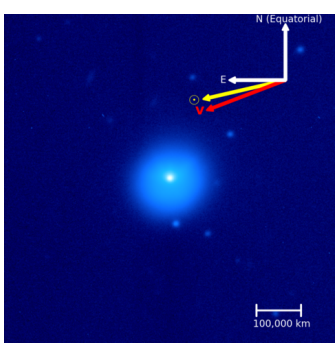

(a) Sept. 25.5

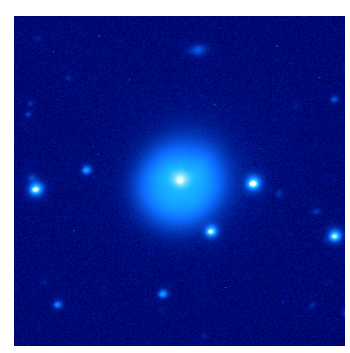

(b) Sept. 26.5

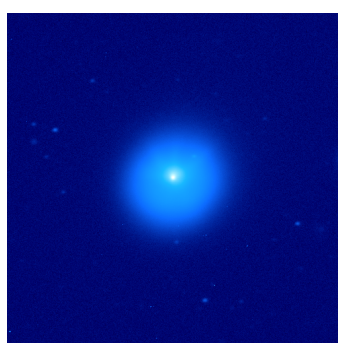

(c) Sept. 27.5

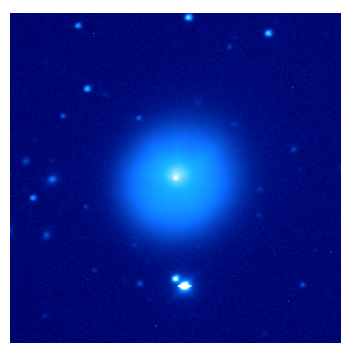

(d) Sept. 28.5

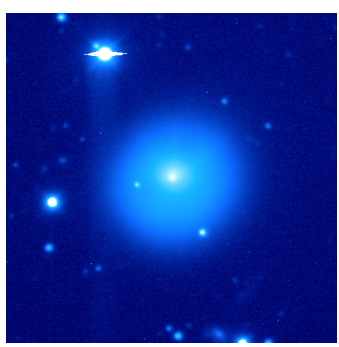

(e) Sept. 29.5

Figure 1: Sample of images from each night of the UT 2008 Sept. observations. Each figure is oriented with equatorial north up and east to the left. A yellow arrow represents the projected direction of the Sun, while a red arrow shows the skyplane projected direction of motion for SW1 during the observations. A scale bar shows the projected distance of 100,000 km at SW1. In each panel, a subsection of $500 \mathrm{x} 500$ pixels of the CCD chip with SW1 at the center are shown. Annotations in (a) are also applicable to images (b)-(e). In each image a projected shell of material forms a nearly symmetric circular coma, which is observed to expand radially during the five nights. Additionally, four linear features are detectable on the northern side of the coma. The enhancement routines bring out the contrast of these features and are shown in Figures $2-4$. 


\section{Image Analysis}

\subsection{Enhancement Routines}

The image enhancement routines used for the 2008 observations are: division by a $1 / \rho$ profile (where $\rho$ is the projected cometocentric distance from the nucleus), application of a radially-varying spatial filter (RVSF), and rotational shift differencing of each image. Each technique has a detailed description in Samarasinha and Larson (2014), which was used by the authors to write image enhancement routines in the programming language Python. These routines are also available online using CometCIEF (Cometary Coma Image Enhancement Facility) through the Planetary Science Institute. Other enhancement routines, which are described in the aforementioned paper, were applied to the 2008 observations, but did not show evidence of structures not seen in the three routines described above.

Figure 2 shows the images after removal of the $1 / \rho$ profile, indicating deviations from a steady mass-loss rate. The shell of material is clearly visible during each night and the projected radial distance of the shell from the nucleus is increasing with time. The shell's projected width is also observed to increase indicating a velocity dispersion of the outflowing dust grains. Figure 3 shows the observations after application of a radially-varying spatial filter. This enhancement brings out brightness variations oriented in both radial and azimuthal directions. Again the expanding shell is seen, but now the four linear features are more pronounced. Lastly, the images were enhanced by rotating them around an axis centered on the nucleus' pixel location by an angle $\pm \theta$. Each rotated image was then subtracted from the original with their differences added together resulting in the images seen in Figure 4 (rotational shift differencing technique). A rotation angle of $\pm 18^{\circ}$ was found to give the most contrast in the enhanced images. This rotation angle resulting in the best enhancement gives a rough scale for the widths of the linear features present. Each linear feature is seen to maintain its PA during the five nights of observation. The lack of curvature observed is unfortunate and restricts the amount of information which can be obtained about the rotational state of the nucleus, but we were able to place constraints on possible rotational states which are discussed in Section 4.

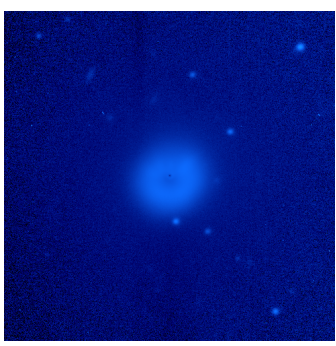

(a) Sept. 25.5

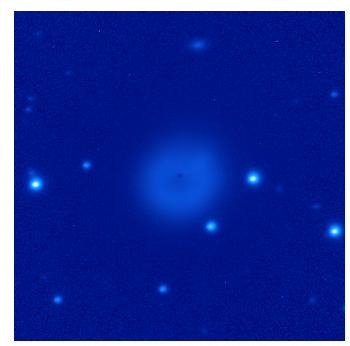

(b) Sept. 26.5

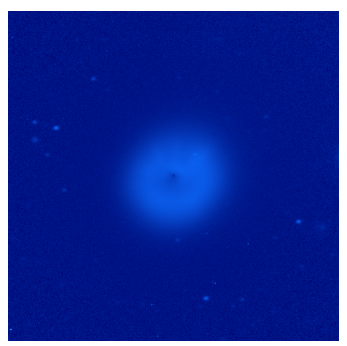

(c) Sept. 27.5

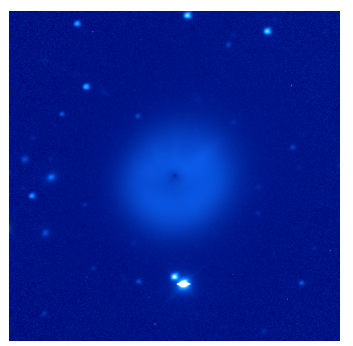

(d) Sept. 28.5

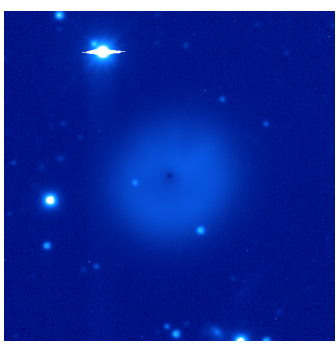

(e) Sept. 29.5

Figure 2: (a)-(e) show enhanced images after the $1 / \rho$ removal. Figures 2(a)-(e) correspond to (a)-(e) in Figure 1, with the earlier figure's orientation and scale applicable here too. The shell of material is readily seen expanded from night to night. This is indicated by a brighter ring structure centered on the nucleus (nucleus is at the center of each panel). The darker area inside of the bright ring indicates the shell-like nature of the outflowing material and that the outburst had ended before the observations ( in contrast, a cone of continuous ejected material would result in no decrease in brightness towards the center of the enhanced image).

\subsection{Shell of Material}

A projected shell of material was observed expanding away from the nucleus. This is best observed from the RVSF-enhanced images shown in Figure 3. Radial profiles from each night of observation reveal the expanding shell 


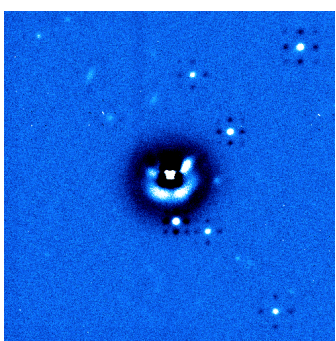

(a) Sept. 25.5

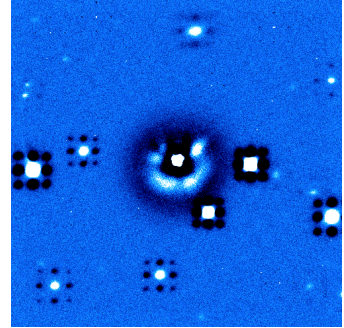

(b) Sept. 26.5

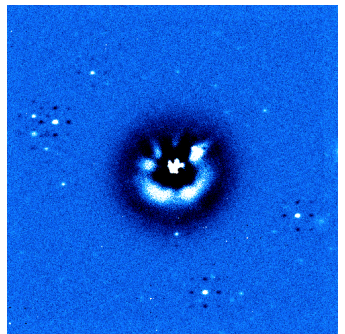

(c) Sept. 27.5

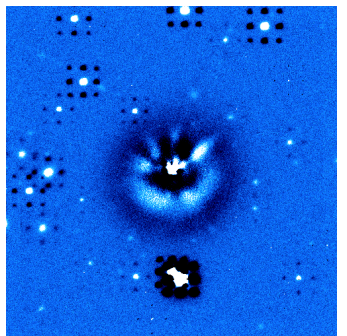

(d) Sept. 28.5

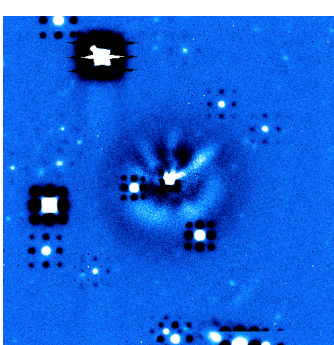

(e) Sept. 29.5

Figure 3: (a)-(e) show a sample of images after application of a radially-varying spatial filter. The orientation and scale of the figure is the same as in Figure 1. The shell of material is seen clearly in the southern region expanding radially during the observations. The dark regions of discontinuity on the northern side of the coma corresponding to the shell are due to the presence of the four linear features. The shell of material is still present in this region, but the linear features dominate due to the characteristics of spatial filtering (Samarasinha and Larson 2014). The dark regions around field stars present in the images are artifacts of the spatial filtering too.

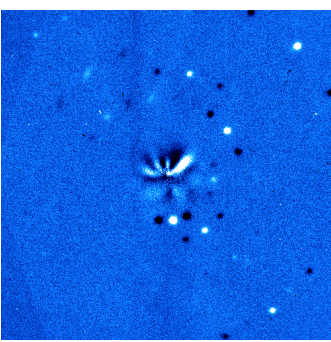

(a) Sept. 25.5

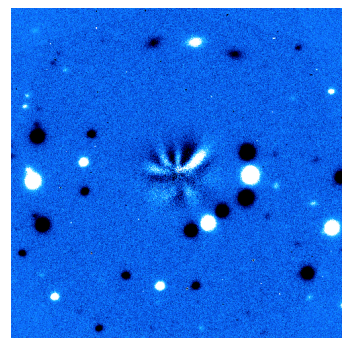

(b) Sept. 26.5

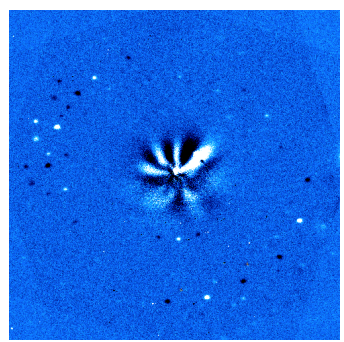

(c) Sept. 27.5

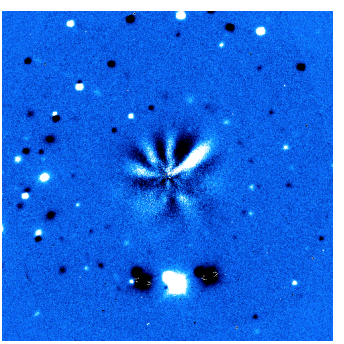

(d) Sept. 28.5

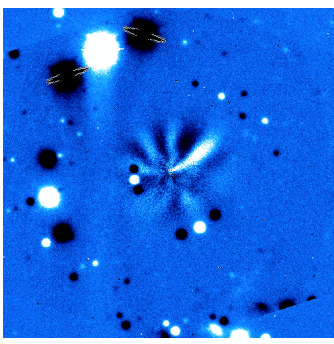

(e) Sept. 29.5

Figure 4: (a)-(e) show a sample of images after application of the rotational shift differencing technique. The orientation and scale of the figure is the same as in Figure 1. The four northern radial features are present in each night's observation, indicating that these features are long lived in the coma. Their position angles $37^{\circ}, 78^{\circ}, 300^{\circ}$, and $353^{\circ}$ are seen not to deviate radially or temporally. Low contrast linear enhancements on the southern side of the coma are also present, but are not as identifiable as those in the north. Most likely these are due to more gradual brightness variations in the shell of material similar to that seen in the north. Corresponding southern linear features in the un-enhanced images could not be found (i.e., they are extremely low contrast), so their incorporation into further analysis is ignored. Artifacts from the rotation-subtraction enhancement result in two dark negative images for each field star. Disappearing of the shell (an azimuthal feature) is also an artifact of this image enhancement technique.

of material. An example is shown for position angles $\mathrm{PA}=140^{\circ}$ and $\mathrm{PA}=230^{\circ}$ in Figure $5(\mathrm{a})$. The peak positions in the RVSF profiles correspond to a higher intensity or peak surface brightness variation in the un-enhanced images (not shown). It is possible that radial positions of the peak brightness variation for each night do not correspond to the same dust grains in each night of observation. Effects such as grain fragmentations and/or grain-brightness fading could mean that the Gaussian peak positions measured from radial profiles correspond to different dust grains during each night of observation. However, it is clear that a shell of expanding material is seen through the five nights of observation. What is unclear is whether the velocity measurements made are actual dust grain velocities or a "group velocity" measurement made of the dust grain size distribution.

To generate the radial profiles, the RVSF images were first "unwrapped" using a $(\rho, \theta)$ polar coordinate conversion. Since each image has been centered on the nucleus's position, $\rho=0$ corresponds to the nucleus. Next, an azimuthal binning of the radial profile was done for a $\Delta \theta=10^{\circ}$. A Gaussian profile was fit to the peak region of each night's profile, 


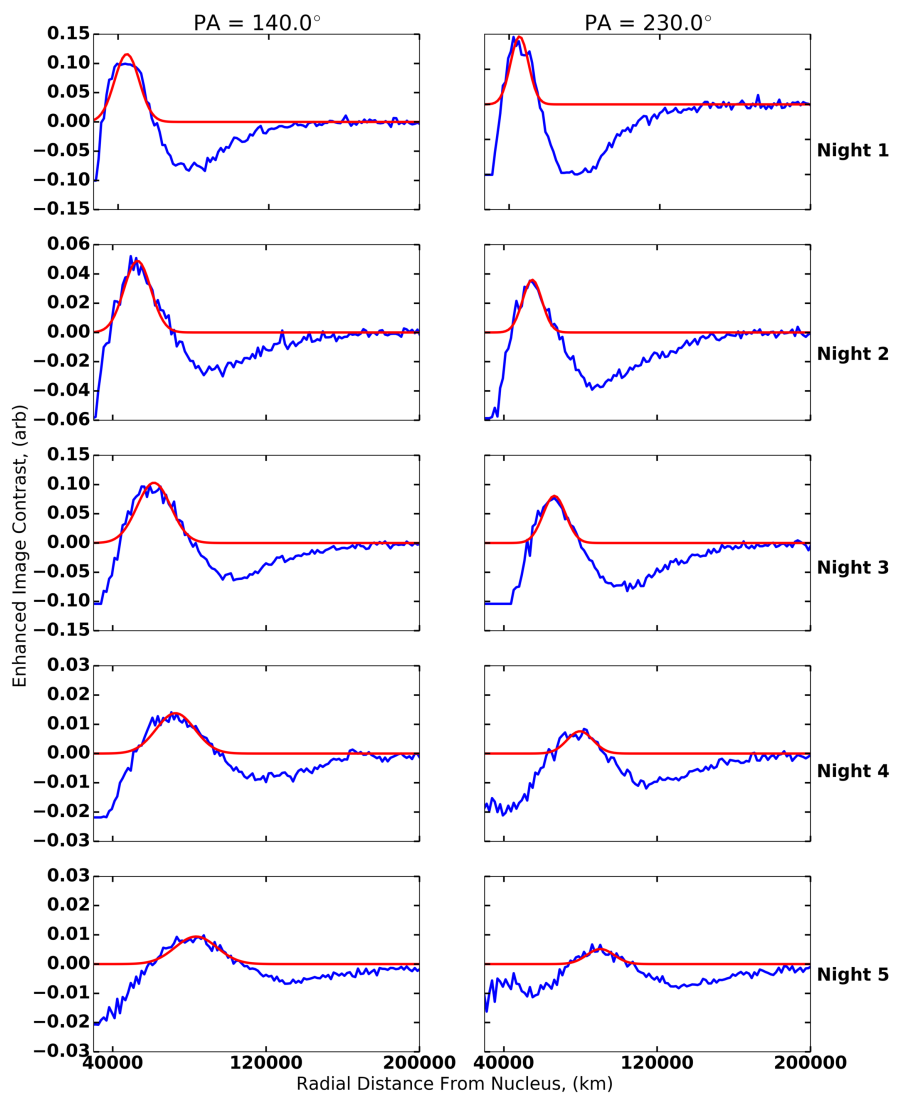

(a)
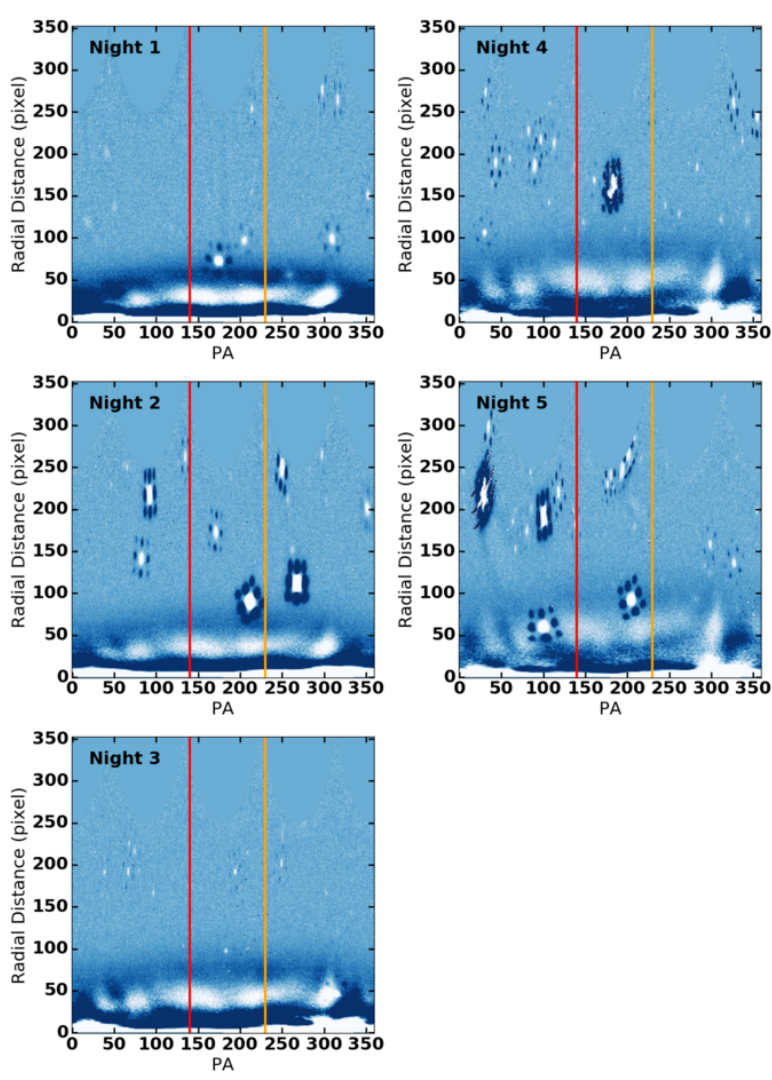

(b)

Figure 5: (a) Radial profiles of RVSF-enhanced images in blue from each night of observations for the position angles $140^{\circ}\left( \pm 5^{\circ}\right)$ and $230^{\circ}\left( \pm 5^{\circ}\right)$. (b) Corresponding unwrapped RVSF images with a red vertical line indicating the position of the PA $=140^{\circ}$ profile and orange line indicating the $230^{\circ}$ profile. The radial profiles clearly show the expanding shell of material. The peak position of the shell was found by fitting the profile with a Gaussian function. The best-fit Gaussian profile in red is also included in each plot. The decrease in relative amplitude of the shell's peak compared to the relative width of the shell over the course of the five nights is attributed to both dust grain size sorting and velocity dispersion of the grains. It should be noted that the RVSF enhancement does not preserve the amplitude of intensity variation of the image, but if the same set of RVSF parameters are used to enhance each image, the positions of intensity variation are preserved. In the unwrapped RVSF images (Figure 3), the shell can be identified as the horizontal white feature seen to move to larger radial positions throughout the five nights of observation. Field stars present in the unwrapped RVSF images are identified by a white spot surrounded by eight dark blue spots.

with the center taken as the shell's position. Figure 5(b) shows an example of the unwrapped RVSF images along with vertical lines indicating the corresponding PAs of the radial profiles in Figure 5(a). The possibility of an asymmetric projected shell expansion velocity was investigated by measuring the shell positions for a range of PAs between $120^{\circ}-250^{\circ}$. Using the Gaussian peak positions, the image pixel scale, and their corresponding observation times, projected velocity measurements were calculated for the expanding shell at each PA. For each PA, a total of ten unique velocity measurements were made using different combinations of peak feature position and their associated time of observation: $v_{i j}=[($ Peak Position $\left.)_{i}-(\text { Peak Position })_{j}\right] / \Delta t_{i j}$, where $i$ and $j$ identify the specific night of observations and $\Delta t_{i j}$ is the time between 
the observations. No significant asymmetry in velocity measurements are observed for different PA positions. The velocity differences from measurements of different PAs was comparable to the scatter in measurements from a single PA. The PA range from $120^{\circ}-250^{\circ}$ allowed 140 independent velocity measurements for the expanding shell. The distribution of velocities was found to follow a Gaussian distribution and is shown in Figure 6. An overall projected velocity value of 0.11 $\pm 0.02 \mathrm{~km} / \mathrm{s}$, using the $1-\sigma$ deviation as the uncertainty for the velocity measurement.

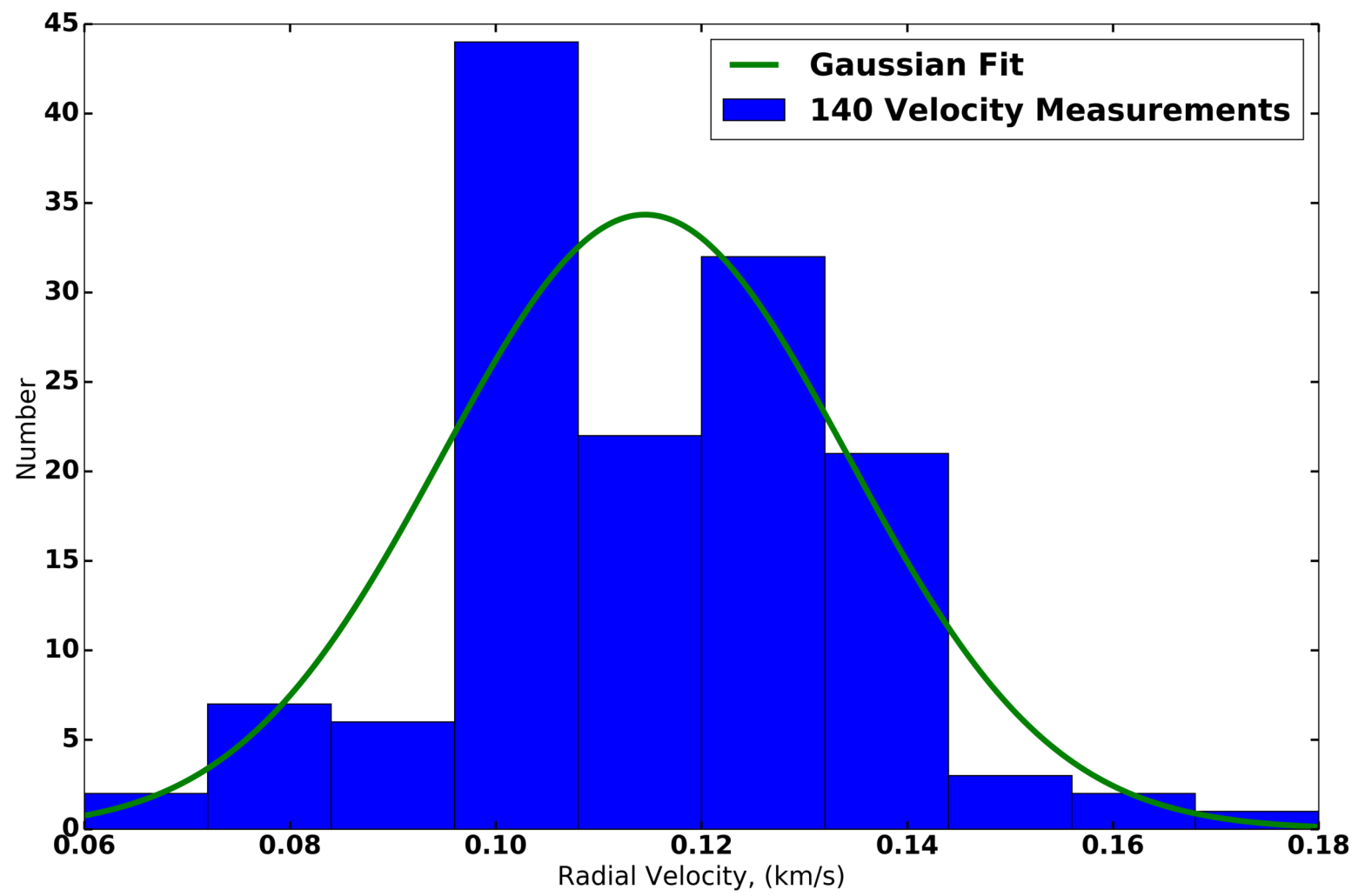

Figure 6: Histogram of the 140 velocity measurements along with a Gaussian model to the velocity measurement distribution. The velocitymeasurement histogram values were fit with a Gaussian. The returned Gaussian mean and standard deviation were used as the outflowing material's velocity measurement: $(0.11 \pm 0.02 \mathrm{~km} / \mathrm{s})$.

Using the velocity measurement and its distribution, a Monte Carlo approach was used to extrapolate and measure the initial time of outburst. The Gaussian peak positions for the five nights of observations were taken to be the distances traveled by the outflowing material since the outburst occurred. The observation time from the FITS headers gave an accurate time stamp for each peak position. The time associated with the middle of the exposure was used for calculations and all images had a $240 \mathrm{~s}$ integration time. For each of the 140 peak-position and timestamp pairs, 1000 velocity values were chosen randomly from the velocity distribution to calculate an outburst start time $\left(\mathrm{UT}_{\text {start }}\right)_{i, j}=\left(\left(\mathrm{UT}_{\text {observation }}\right)_{i}-(\text { Peak Position })_{i} / v_{j}\right)$, where $i$ refers to the 140 pairs and $j$ refers to the 1000 velocity values. The value of 1000 was chosen to sample the velocity distribution well, producing a distribution of outburst start times following a Gaussian distribution. Using this method, a date of 2008-09-21.03 \pm 0.95 days was found for the beginning of the outburst. 


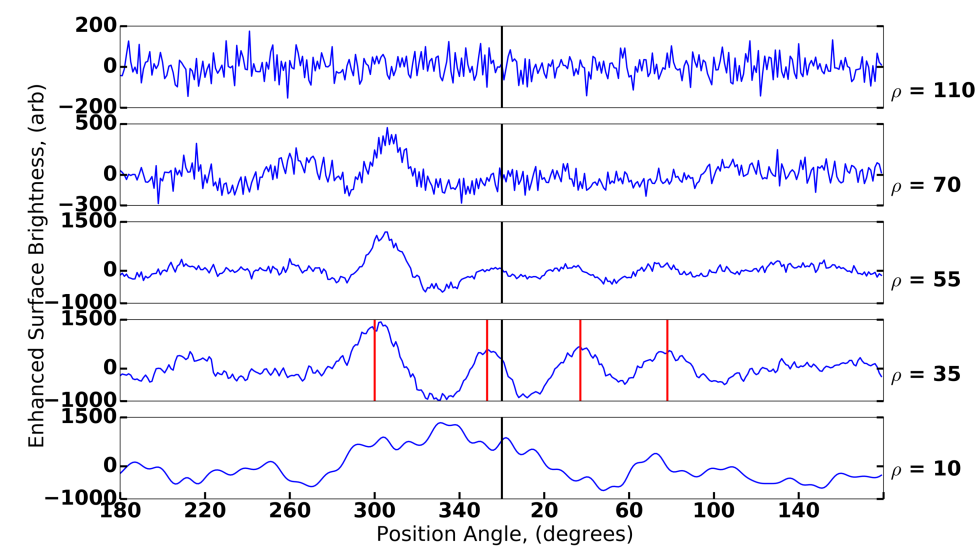

(a)

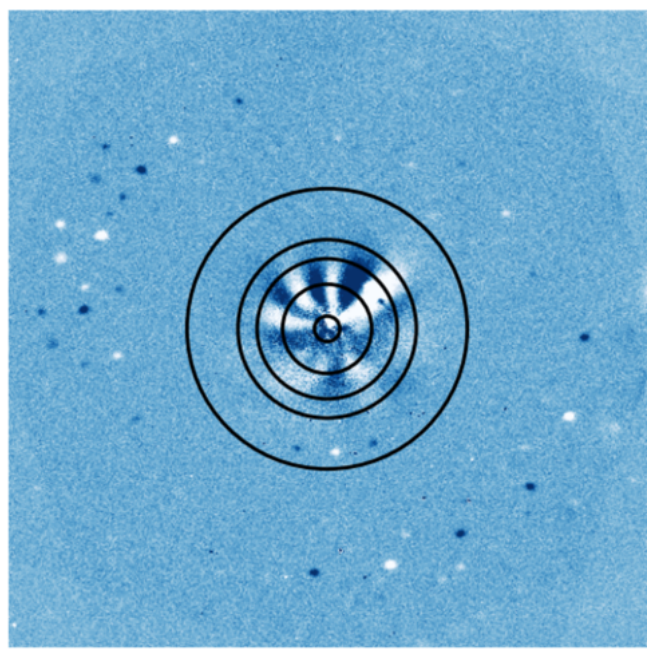

(b)

Figure 7: (a) Azimuthal profiles from the rotational shift differenced image from the third night of observations. The PA $=0^{\circ}$ has been shifted so that it is in the middle of the plots and is indicated by the black vertical line. The radii of the profiles, indicated by the projected radial distance value on the right of each panel in terms of image pixels (pixel scale is $1415.7 \mathrm{~km} / \mathrm{pixel}$ ), are such that at least one profile is interior to and one exterior to the expanding shell of material. The four linear features appear as bright bands in the enhanced images and their corresponding peaks are observed in the profiles indicated by the red vertical lines in the $\rho=35$ panel. The red vertical lines identifying the linear features were omitted in the remaining panels to improve the visibility of the plots. The rotationally enhanced image from the third night of observations is shown in (b) along with a mask overlaying the positions of the profiles. It can be clearly seen that the outer profile does not contain any signatures of the linear features. The inner profile is a little trickier, where close visual inspection of the image suggests that the features are possibly connected with the nucleus and influenced by the specific $\pm 18^{\circ}$ rotational shift. Figure 7 (b) has the same orientation and scale as Figure 1.

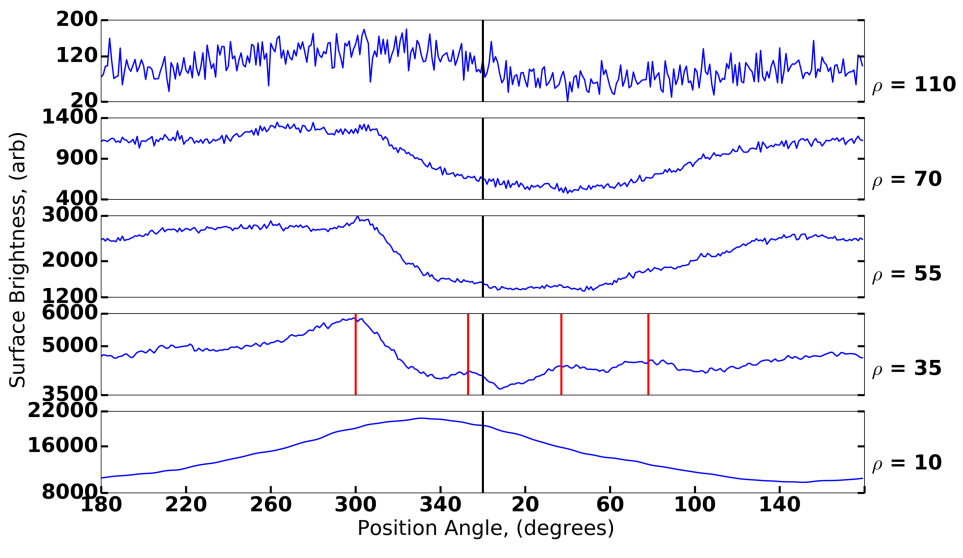

(a)

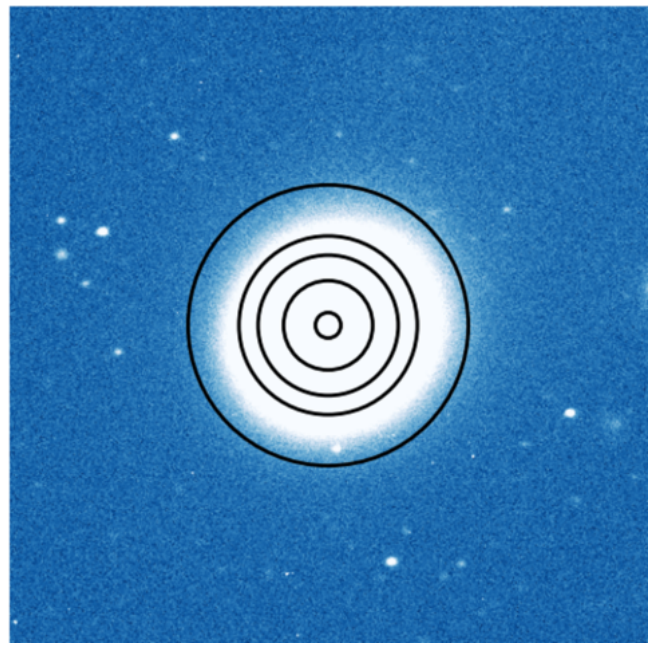

(b)

Figure 8: Similar to Figure 7, but for the corresponding un-enhanced image from the third night of observations. (a) Azimuthal profiles and (b) corresponding masked image. Investigation of (a) shows the same four regions in the un-enhanced images as having an increase in surface brightness. What is more pronounced in the images is the containment of the linear features in the shell of material, consistent with our assessment of the features being part of the outburst and not from separate active areas or events. 

Monte Carlo coma simulation.

\section{Monte Carlo Coma Modeling}

Morphological features in a comet's coma have proven useful for placing constraints on properties of cometary nuclei which may be obscured from direct observation by the coma itself (Sekanina and Larson 1984; Schleicher and Woodney discern the rotation state and nucleus surface area(s) of activity. The dynamic features measurable from observations are highly dependent on their timescales and on the cadence of observations. 3-D Monte Carlo coma modeling is used to measure or constrain properties of the nucleus' spin state by generating synthetic comet images and comparing them with observations. In this section we describe the 3-D Monte Carlo coma model (Samarasinha 2000; Samarasinha and Larson

The linear features seen in the north of the coma are best observed in the rotational shift differencing enhanced images (Figure 4). Four very pronounced features are seen to expand radially throughout the five nights of observations. The position angles of the features are $37^{\circ}, 78^{\circ}, 300^{\circ}$, and $353^{\circ}$. No major curvature is seen in the features indicating a possibly slow rotation period and/or short outburst duration. By analyzing the linear extent of the radial features it was the same event as the expanding shell. Figure 7 shows azimuthal profiles for an array of radial positions for a sample rotationally shift differenced image from the third night of observations. The radial positions for the profiles where chosen so one profile would be interior to the shell's inner boundary, three contained within the shell's radial boundaries, and one exterior to the shell's outer radial boundary. These positions verified the inner and outer boundaries of the four linear

The azimuthal profiles for the rotationally-shift differenced image in Figure 7 show that the linear extent is within the shell. It is important to note again the tendency of the enhancement routines to generate artifacts in the resulting images. One must analyze any feature produced through enhancements and verify its corresponding feature in the un-enhanced image. Figure 8 shows the same azimuthal profiles as in Figure 7, but for the un-enhanced image. While subtle, the four peaks can be seen for the three inner azimuthal profiles.

\subsection{Inferred Outburst Coma Structure}

The coma morphology and its evolution over the five nights suggest its 3-D structure resembles a cone of outflowing material probably projected towards the general direction of the observer. The semi-circular shape of the coma seen in observations is due to the projection of the 3-D cone onto the sky plane. With a source region on the nucleus' surface slightly south of the sub-Earth point and an initial material ejection direction slightly south of the sub-Earth vector direction, the projected cone of material generated by this geometry explains the asymmetric circular shape seen in the observations. With the expanding material producing a projected nearly circular ring around the nucleus, the location of the outburst on the surface can be placed close to the sub-Earth point on the nucleus. It should be mentioned that the solar phase angle during observations is small, $\approx 10^{\circ}$ (i.e. source region for the outburst is close to the peak insolation which is also close to the sub-Earth point on the surface of SW1). In the next section we build on this by using a 3-D 2003; Farnham 2009, Samarasinha et al. 2011; Mueller et al. 2013). By tracking features in the coma, it is possible to 2014) used for modeling the SW1 2008 outburst and show constraints derived for the nucleus of SW1. 
3-D Monte Carlo Coma Model Input Parameters

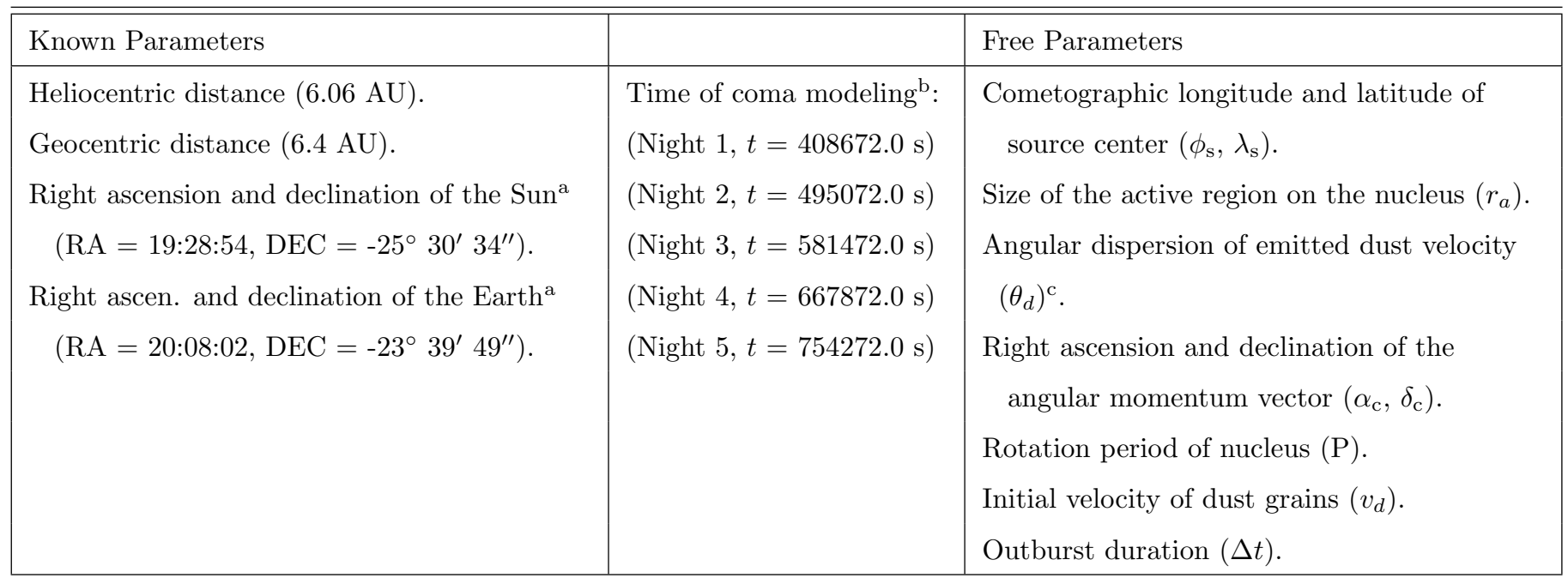

a RA and DEC as viewed from SW1 at the time of the observations.

b $t=0$ refers to the beginning of the outburst.

c Angular dispersion of the initial dust grain velocity direction, with $0^{\circ}$ being normal to the local surface plane.

The coma model assumes a nucleus with active areas represented by circular regions on the surface. No shape information for SW1's nucleus is known so nucleus shape is ignored during modeling. A principal-axis (PA. It should be noted that PA is also used to refer to position angle is this paper.) rotation state (Samarasinha et al. 2004) was initially used for modeling due to recent measurements of SW1's nucleus size being larger when compared to other JFCs $(R \sim 27$ km, Stansberry et al. 2004; $R \sim 19$ km, Stansberry et al. 2009; $R \sim 23$ km, Fernández et al. 2012; $R \sim 30$ km, Schambeau et al. 2015). Although other measurements have indicated non-principal-axis (NPA) rotation for SW1's nucleus (Meech et al. 1993), SW1's larger size gives confidence in the PA assumption. Damping forces for a larger nucleus in an excited state of rotation (NPA) would quickly result in a PA state (Samarasinha et al. 2004). Also, torques driven by outgassing, outbursts, and micrometeorite impacts are relatively ineffective at disturbing the rotation state because of the relatively larger nuclear size.

Table 2 shows input parameters for the coma modeling and their associated modeling values. Free parameters are identified by their associated symbols. The generation of an outburst coma model proceeds as follows (note: the term "coma" or "coma model" used here refers to the portion of SW1's coma generated by the outburst event and excludes the quiescent background coma unless explicitly stated). The orbital geometry of the observations is set up for the specific night of observation. A set of input values for the free parameters are chosen and the Monte Carlo modeling code is run for the appropriate time of the given night under investigation. Once the 3-D coma model has been generated, it is projected onto the sky plane of the observations, thus generating the synthetic coma observation. It should be noted that the coma modeling routine only generates the portion of the coma produced by the outburst. To fully generate a synthetic comet image, flux contributions from the nucleus and background (quiescent) coma must be added to the outburst coma model. 
The background coma was modeled using a canonical $\frac{A}{\rho}$ profile, where $A$ is a scaling factor to match the background coma's flux. To generate the final synthetic comet image the point spread function (PSF, simulated with an Airy function), outburst coma, and quiescent coma are added together, then convolved with an appropriate kernel to mimic the seeing conditions of the observations.

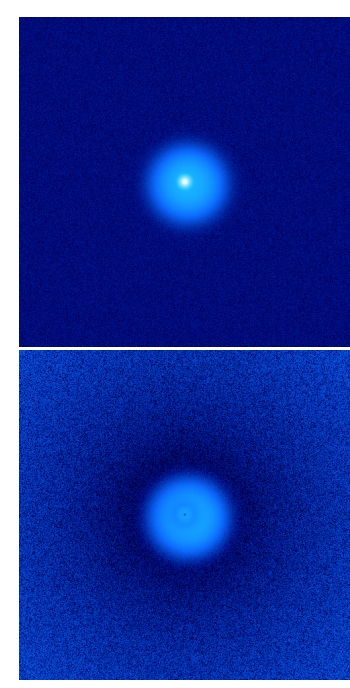

(a) Sept. 25.5
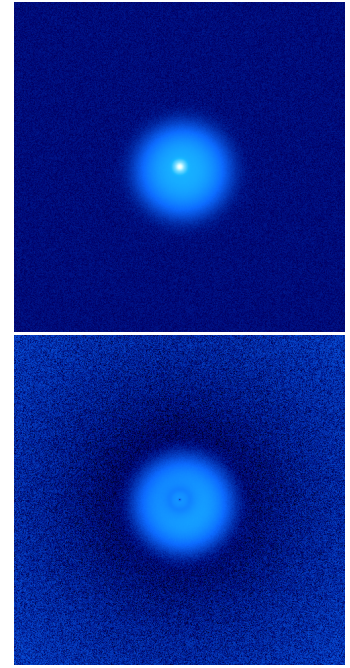

(b) Sept. 26.5
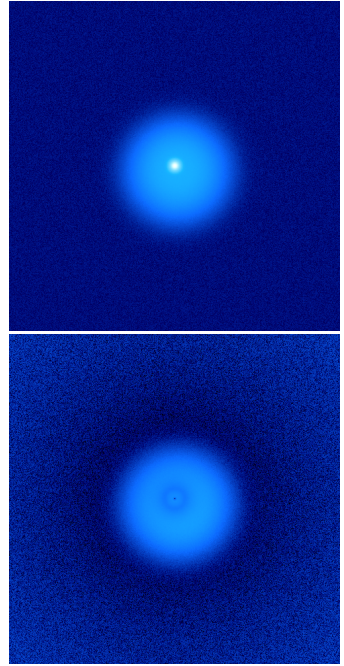

(c) Sept. 27.5

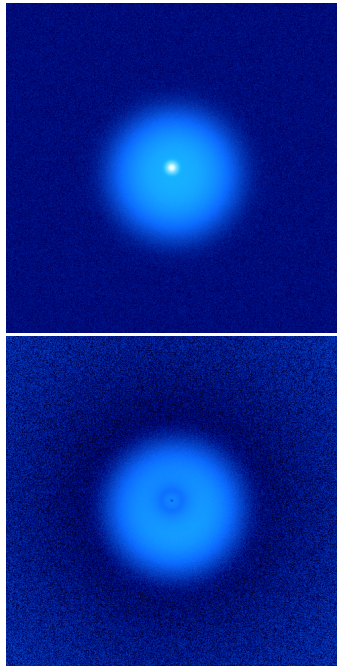

(d) Sept. 28.5

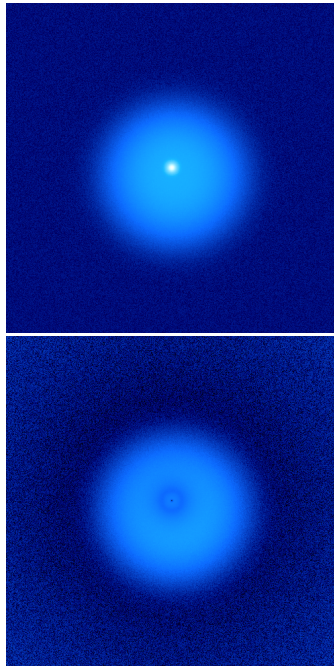

(e) Sept. 29.5

Figure 9: Top row: (a)-(b) Synthetic comet images for the five nights of observations. Using an active area covering $\sim 6 \%$ of the surface and a large $\mathrm{P} / \Delta t$, the synthetic comet images are able to reproduce morphology seen in the observations. The expanding shell is accurately reproduced, with a slight offset of the nucleus to the north of the shell. The images have the same pixel scale, image scale, and orientation as Figure 1. Bottom row: Same synthetic comet images from the top row, but with a $1 / \rho$ removal similar to Figure 2 . The removal of the $1 / \rho$ profile brings out the outburst coma shell in the synthetic models.

\subsection{Nucleus Spin State Results}

Initial analysis of the five nights of observation resulted in the realization that the determination of a spin-pole orientation is not possible with this data set. The morphological features present in the observations do not suggest any sense of rotation (e.g. curved features characteristic of a rotating nucleus). The similarity present throughout the five nights signifies an outburst duration that is significantly shorter than the rotation period. If the outburst duration was a significant fraction of the rotation period, then signatures of dust ejection over an angular arc would be present. The coupling between the spin period and outburst duration impacts the analysis of the coma morphology because features present in observations can be attributed to either property. This analysis can only result in constraints on the spin period to outburst duration ratio $(\mathrm{P} / \Delta t)$. While we can't say definitively what the spin period and pole orientation are, we can still constrain the spin period for assumed spin-pole orientations.

Certain properties of the outburst inferred from the observations are valid for any spin-pole orientation (e.g. the active area's size and center location with respect to the sub-Earth point on the nucleus' surface). To place a constraint on these parameters, the rotation period was fixed to 10,000 days, so that any morphological features in the coma due to nucleus rotation are negligible and the outburst duration was set to 10.5 hours. Using the cone-of-ejected-material hypothesis (Section 3), the location of the source center was found. A grid of source region locations was setup to sample 
the parameter space well. Each set of parameters was used to generate a set of synthetic comet images representing one image for each of the five nights of observation. Comparing the synthetic comet images with observations resulted in a best-fit set of parameters. A source region having a $28^{\circ}$ angular radius ( $\sim 6 \%$ of the surface) and location $\sim 4^{\circ}$ below the sub-Earth point most suitably reproduced the expanding shell of material. It was found that a $0.3 \mathrm{~km} / \mathrm{s}$ initial dust grain velocity, including a $10^{\circ}$ angular dispersion of velocities with respect to the local surface normal direction, generated the $0.11 \mathrm{~km} / \mathrm{s}$ projected shell of expanding material. It should be noted that other initial dust grain velocities combined with other shapes for the 3D outburst coma structure (e.g. hemispherical as opposed to conical) can also result in the $0.11 \mathrm{~km} / \mathrm{s}$ measured projected shell expansion velocity. The above stated $0.3 \mathrm{~km} / \mathrm{s}$ scenario fit the observations more closely when comparing radial surface brightness profiles and thus was chosen as the "best-fit" solution. Figure 9 shows the synthetic comet images resulting from the best-fit parameters for each of the five nights of observation. The linear features in the northern region of the coma have been ignored during this stage of modeling since they are simply a product of higher dust grain density regions. No independent or additional information would be gained from their inclusion in this stage of modeling.

Effects due to solar radiation pressure have been ignored during Monte Carlo coma modeling. The region of the outburst coma observed and modeled is in the inner coma region where solar radiation pressure has not significantly altered the emitted dust grain's sky-plane velocity. This was determined by calculating the distance $d$ traveled by a dust grain in the projected sky-plane of the observations before being turned back due to solar radiation pressure (Mueller et al. 2013).

$$
d=\frac{v^{2} \cos \gamma^{2}}{2 \beta g \sin \alpha}
$$

where $v$ is the initial ejection velocity of the dust grains, $\gamma$ is the angle between the initial direction of the dust grains and the sky-plane, $\beta$ is the ration of radiation pressure acceleration to solar gravity, $\alpha$ is the solar phase angle of the observations, and $g=G M_{\odot} / R_{h}^{2}$ is the gravitational acceleration on the dust grains. Using $\beta$ values of $(0.1,0.6$, and 1.0$)$ we calculate values for the projected turn-around distance of $d \approx(3,500,00 \mathrm{~km}, 590,000 \mathrm{~km}$, and 350,000 km) for dust grains leaving the edge of the modeled source region (i.e. a value of $\gamma=\left(90^{\circ}-28^{\circ}\right)=62^{\circ}$ ). The projected sky-plane radius of the coma's shell for the fifth night of observations is less than 100,000 km, as seen in Figure 1, signifying radiation pressure effects have not significantly altered the coma's morphology.

With constraints found for the active area's location and size, dust grain velocity, and dust grain velocity angular dispersion it is now possible to place constraints on the spin period for a set of assumed spin-pole orientations. As was stated earlier, the morphology of the observations did not have sufficient structure with differentiating characteristics to determine the spin-pole orientation, so making assumptions on the orientation allows constraints to be placed on the spin period for these specific orientations. This information will be useful in future analysis of SW1 outburst observations and to provide possible starting points for analysis of different outburst observations. Four spin-pole orientations were chosen: perpendicular to the Sun-Earth vector during observations $\left(\mathrm{RA}=300^{\circ}, \mathrm{DEC}=66^{\circ}\right)$, in the same plane as the Sun-Earth vector, but perpendicular to the Earth direction during observations $\left(\mathrm{RA}=32^{\circ}, \mathrm{DEC}=0^{\circ}\right), 45^{\circ}$ above the Sun-Earth plane and $45^{\circ}$ between the spin-pole direction and the Earth direction $\left(\mathrm{RA}=347^{\circ}, \mathrm{DEC}=21^{\circ}\right)$, and along the Earth direction $\left(\mathrm{RA}=302^{\circ}, \mathrm{DEC}=-23^{\circ}\right)$. The procedure for placing a constraint on the spin period is similar for each pole orientation. First, the modeling code input parameters were changed to reflect the relevant spin-pole orientation. Next, 
the spin period was set to 10,000 days and outburst duration set to 10.5 hours, with the modeling code run for each of the five nights of observation. This gave a baseline for comparing the images when decreasing the spin period. The spin period was then decreased until the synthetic comet images deviated significantly in morphological structure when compared with the observations. Figures 10 and 11 summarize the results of the spin period analysis for the four assumed outburst require
Earth direction.

\subsection{Dust Velocity Distribution and Outburst Duration} brightness (SB) profiles of the comet observations provided this independent method of analysis. Longer duration outbursts result in more dust grains closer to the nucleus and thus steeper slopes for the radial SB profiles in the synthetic comet images. Plots of radial SB profiles for a selection of four PAs from the comet observations and the synthetic comet images were compared to place constraints on the outburst duration. The synthetic comet images are similar to those from Section 4.1 and have been convolved with a Gaussian kernel to mimic the seeing conditions during the observations.

The velocity distribution of the Monte Carlo coma model was varied to see its effect on the modeling output. First, a boxcar distribution for velocity was used to mimic a delta function where only the initial velocity $\left(v_{o}\right)$ was chosen. A random initial angular deviation from the local surface normal was used to impart dispersion in the velocities. A dispersion of $\theta_{d}=10.0^{\circ}$ was used for all modeling. Secondly, a Gaussian distribution with a std. deviation $\sigma$ of $\pm 0.2 v_{o}$ in velocities the spin-pole orientation equivalent to the top of Figure 10 was assumed for the nucleus for both distributions. Figure 11 shows a sample of radial SB profiles from both distributions. It was found that a Gaussian velocity distribution more accurately modeled the observations. It should be noted that the modeling shown in Section 4.1 assumed a Gaussian velocity distribution identical to that used in the models generating the radial SB profiles in Figure 11(b).

Using the Gaussian velocity distribution, the outburst duration was varied to find constraints on its length. Figure 12 shows examples of two outburst durations and how they affect the radial SB profiles of the synthetic comet images. A 5-day outburst (Figure 12(a)) results in too much dust close to the nucleus and thus radial SB profile slopes too steep to model observations. The overall shapes of these profiles also do not match the observations. By decreasing the outburst duration by 0.5 -day steps, an upper limit of 1.5 days was concluded for the length of the outburst. Figure 12(b) shows overall shape matches the observations well. A rescaling of the outburst coma flux, when compared to the nucleus and background coma, is able to shift the profile to match the observations. The offset in between profiles was retained in the figures to remain consistent by applying the same linear scaling of the synthetic comet images throughout this work. Therefore, a 1.5-day outburst duration was chosen as the upper limit.

Decreasing the outburst duration below 1.5 days unfortunately did not result in a lower limit for the outburst duration. All modeled outburst durations below the upper limit generated synthetic comet images that were consistent with obser- 


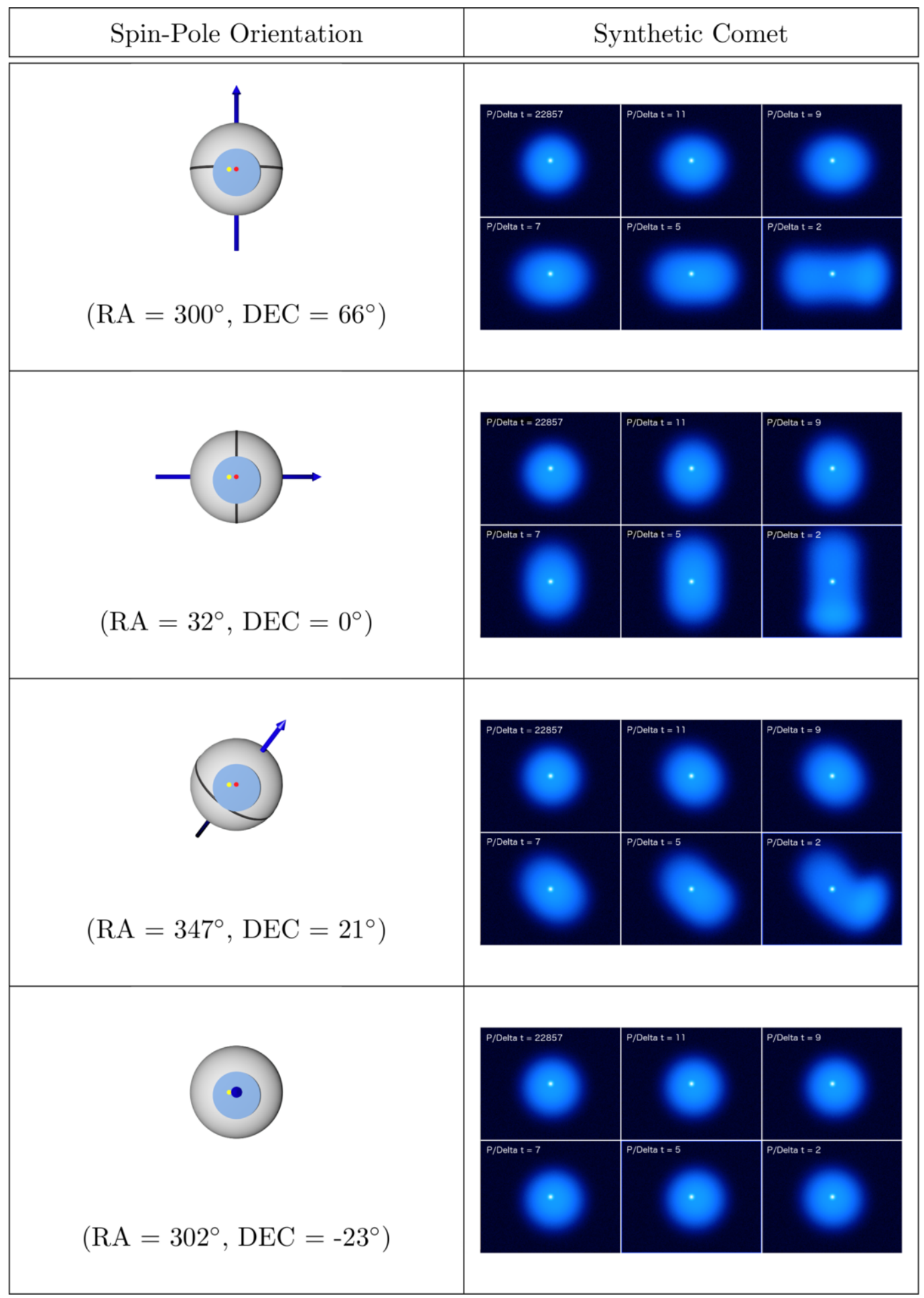

Left: Diagram of spin-pole orientation: blue arrow indicates spin-pole direction, black line represents comet equator, blue circular area indicates the active area on nucleus, red circle indicates sub-Earth point, and yellow circle indicates sub-solar point.

Right: Synthetic comet images for the fifth night of observation for the assumed spin-pole orientation shown to the left. The orientation of the synthetic comet images is the same as in Figure 1. The results are expressed in terms of the ratio of spin period (P) to outburst duration $(\Delta t)$ : (left to right and top to bottom: $22857,11,9,7,5,2$ ).

Figure 10: Spin period constraints assuming possible spin-pole orientations. 

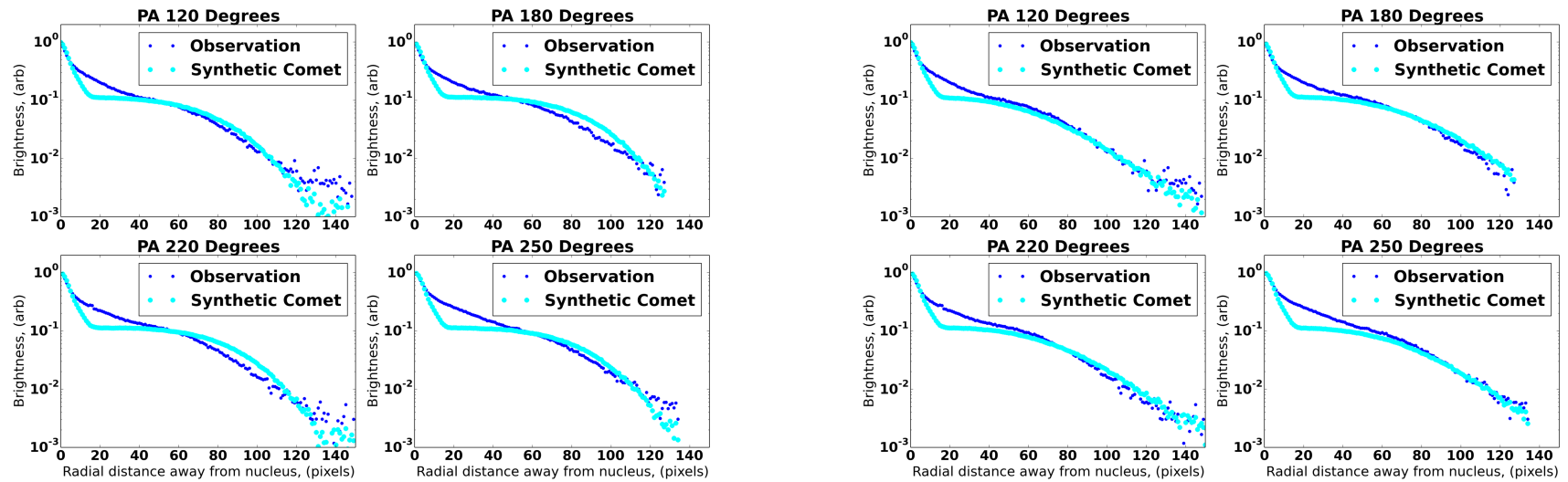

(a)

(b)

Figure 11: Radial SB profiles from the fifth night of observations for a boxcar vs. Gaussian velocity distribution for dust grains. (a) The boxcar velocity distribution radial SB profiles do not model the observations well. The outflowing shell of material is too concentrated in radial direction thus creating the overly curved radial SB profiles when compared to observations. (b) The Gaussian distribution modeled the observations well in the radial direction, ( $\rho \sim 40$ to 140 pixels). The deviation of model and observations in the radial region from $\rho=15$ to 35 pixels is due to the canonical $1 / \rho$ background coma not modeling the observations well and was ignored because of its irrelevance to the focus of this work.

5-day Outburst Duration
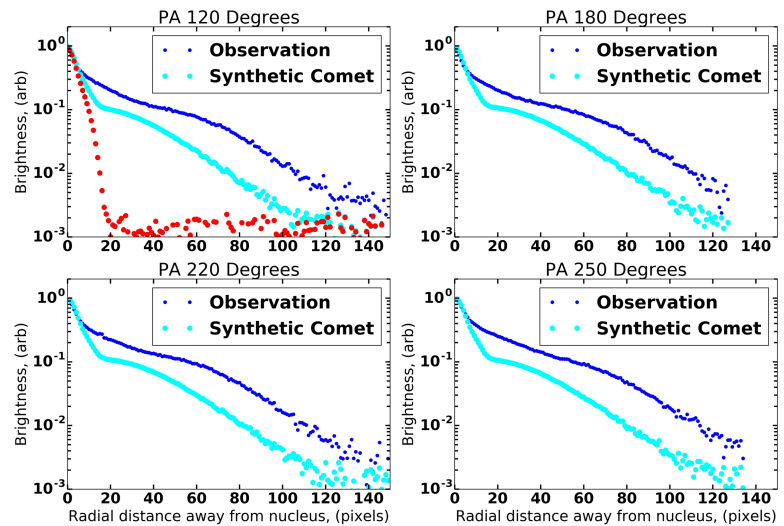

(a) 1.5-day Outburst Duration
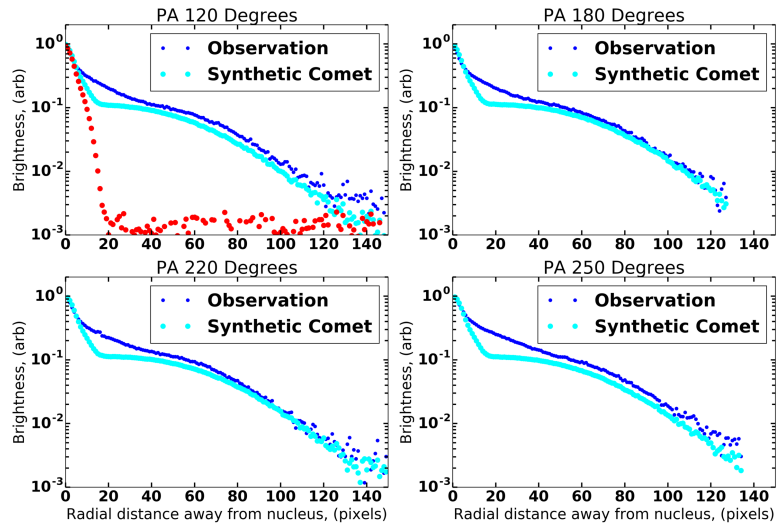

(b)

Figure 12: Radial SB profiles from the fifth night of observations comparing the modeling length of the outburst duration. (a) A 5-day outburst shows deviations between the observation and modeling images. For this situation too much dust is still in the close vicinity of the nucleus during observations. The increased dust number density close to the nucleus leads to the radial SB profiles having a slope too steep. (b) A 1.5-day duration is shown and found to be an upper limit for the outburst duration. Longer outbursts show deviations from the observed radial SB profiles similar to those seen in (a). The red profile in the top-left panel of (a) and (b) represents the comet nucleus with only a canonical $1 / \rho$ profile. 
Summary of Photometry: $R$ Mag, Af $\rho, \dot{M}_{\text {dust }}, M_{\text {dust }}$

\begin{tabular}{lcccc}
\hline \hline Epoch $^{\mathrm{a}}$ & $\begin{array}{c}R \mathrm{Mag}^{\mathrm{b}} \\
\left(10^{\prime \prime}\right)\end{array}$ & $\begin{array}{c}A f \rho\left(10^{\prime \prime}\right) \\
(\mathrm{cm})\end{array}$ & $\begin{array}{c}\dot{M}_{\text {dust }}\left(10^{\prime \prime}\right) \\
(\mathrm{kg} \mathrm{s}\end{array}$ & $\begin{array}{c}M_{\text {dust }}\left(30^{\prime \prime}\right)^{\mathrm{c}} \\
(\mathrm{kg})\end{array}$ \\
\hline 25.5 & $11.9 \pm 0.1$ & $77000 \pm 7000$ & $4700 \pm 500$ & $(1.8 \pm 0.2) \times 10^{9}$ \\
26.5 & $12.0 \pm 0.05$ & $67000 \pm 3000$ & $4100 \pm 300$ & $(2.0 \pm 0.1) \times 10^{9}$ \\
27.5 & $12.2 \pm 0.1$ & $54000 \pm 5000$ & $3300 \pm 400$ & $(1.8 \pm 0.2) \times 10^{9}$ \\
28.5 & $12.5 \pm 0.1$ & $44000 \pm 6000$ & $2700 \pm 400$ & $(1.7 \pm 0.2) \times 10^{9}$ \\
29.5 & $12.6 \pm 0.1$ & $40000 \pm 3000$ & $2400 \pm 200$ & $(1.7 \pm 0.1) \times 10^{9}$ \\
\hline
\end{tabular}

${ }^{a}$ Day of 2008 September, UT at beginning of observations.

b Magnitude measurements are in agreement with analysis of independent observations of the same UT Sept. 2008 outburst (Trigo-Rodriguez et al. 2010).

c Lower limit for total mass of dust emitted during the outburst using a photometric aperture of 30" (using Equation 3 ).

vations. The dispersion in the dust grain velocities becomes the dominant parameter controlling the projected material's radial SB profiles at this limit and not dictated by the spread of material due to an extended period of dust emission.

\section{Dust Production During the Outburst}

\subsection{Outburst Dust Mass Measurement}

Until this point no mention of apparent flux calibration has been made for the 2008 SW1 observations. For the analysis of coma morphology only changes in relative brightness were necessary. Therefore making additional relative flux measurements are appropriate to estimate lower limits for the mass of dust emitted during the outburst. For this,

each image was calibrated with respect to a set of standard stars. For apparent flux calibration we used the outburst observations of SW1 and Sloan Digital Sky Survey (SDSS) r-band magnitudes of stars in each observation frame. Each of the seventeen observations of SW1 from the five nights of observations were calibrated. Aperture photometry of the calibrated images resulted in apparent R-band magnitudes, Af $\rho\left(10^{\prime \prime}\right)$ values, and dust production rates derived from the Af $\rho$ values $\left(\dot{M}_{\text {dust }}\left(10^{\prime \prime}\right)\right)$ for the five nights of observations are shown in Table 3 . The value for each single night is the average of the individual exposures from that night. 


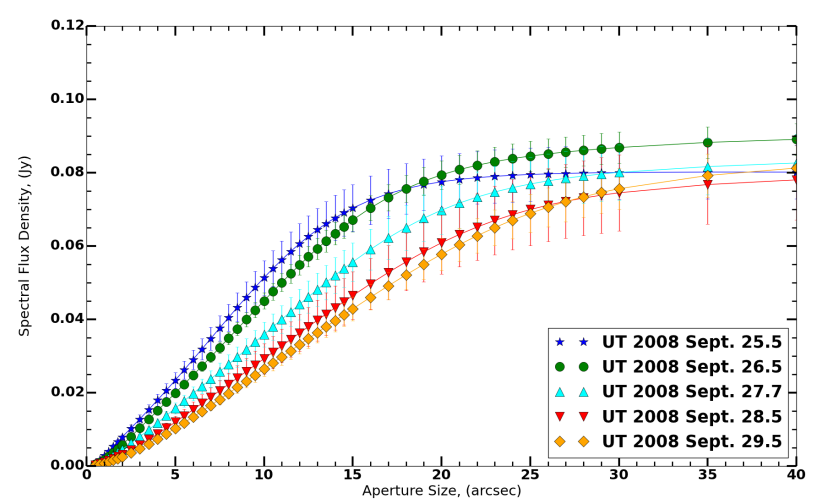

(a)

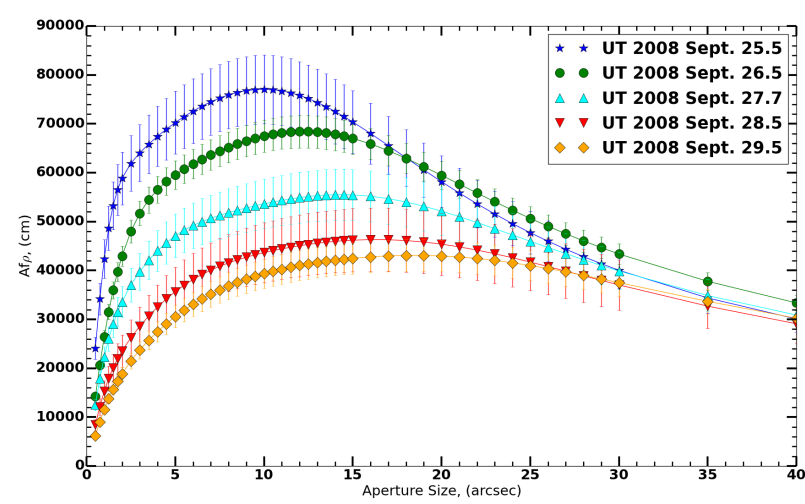

(b)

Figure 13: Profiles from photometry of the five nights of observations. The average photometry values from each night's individual observations were chosen to represent the activity level for that particular night. (a) Spectral flux density measurements from the five nights for an increasing aperture size, where the spectral flux density is a measure of the energy transfer rate of electromagnetic radiation reflected by the dust grains received by the CCD for a specified wavelength. The slopes of the profiles decrease during the five nights representing the movement of dust grains and possibly grain fading and/or fragmentation. For each of the profiles an aperture of $\approx 30^{\prime \prime}$ signifies enclosing all of the flux associated with the comet. For this reason the $30^{\prime \prime}$ aperture flux value was chosen for measurements of the total dust emitted during the outburst. (b) Af $\rho$ measurements for the five nights of observations for the same set of apertures shown in 14(a).

The Af $\rho$ parameter is a proxy for cometary activity first derived in A'Hearn et al. 1984.

$$
A f \rho(\mathrm{cm})=\frac{\left(2 \Delta R_{H}\right)^{2}}{\rho}\left(\frac{F_{\mathrm{com}}}{F_{\odot}}\right)
$$

where, $\Delta(\mathrm{AU})$ is the geocentric distance of the comet, $R_{H}(\mathrm{~cm})$ is the heliocentric distance of the comet, $F_{\text {com }}$ is the observed flux of the comet within a circular aperture of projected radius $\rho(\mathrm{cm})$, and $F_{\odot}$ is the solar flux at 1 AU. Af $\rho$ is derived assuming a spherically-symmetric dust emission and provides an estimate for the dust production rate, $\dot{M}_{\text {dust }}$.

$$
\dot{M}_{\text {dust }}=\frac{8 a \rho_{d} v_{d}(A f \rho)}{3 p}
$$

where, $a$ is the dust grain radius (assumed spherical dust grains of a single size), $\rho_{d}$ is the dust grain density, $v_{d}$ is the dust grain expansion velocity, and $p$ is the geometric albedo of the dust grains. The following standard values were chosen to be consistent with other comets: $a=1.0 \mu \mathrm{m}, \rho_{d}=1000.0 \mathrm{~kg} / \mathrm{m}^{3}$, and $p=0.04$ (Hosek et al. 2013; Ivanova et al. 2011). While there is clearly a dust grain size distribution associated with cometary outburst, the assumed $1 \mu \mathrm{m}$ grain is chosen due to its efficiency at scattering light at R-band wavelengths. It should also be noted that the albedo value used for the dust production rate measurement was adjusted for phase effects of the nonzero phase angle observations of SW1. From this equation the dust production rate measurements were calculated for the $10^{\prime \prime}$ aperture for each of the five nights and are given in Table 3 .

Figure 13a shows the spectral flux-density measurements for increasing photometric aperture size. An $\sim 30^{\prime \prime}$ aperture contains almost all of the comet's detectible flux from the outburst and is used for calculating the total amount of dust mass $M_{\text {dust }}$ ejected during the outburst. Using the same assumptions for the $1.0 \mu \mathrm{m}$ dust grain's size and density as above, 
a lower-limit on the amount of dust emitted during the outburst was calculated with the following equations (Jewitt 1991):

$$
M_{\mathrm{dust}}=\frac{4 \rho_{d} a C_{\mathrm{dust}}}{3}
$$

where $C_{\text {dust }}$ is the total scattering cross section of the collection of $1.0 \mu \mathrm{m}$ dust grains which is measured from the flux contained in the $30^{\prime \prime}$ aperture. $C_{\text {dust }}$ was found with (Lamy et al. 2004):

$$
A \Phi(\alpha) C_{\text {dust }}=2.238 \times 10^{22} R_{H}^{2} \Delta 10^{0.4\left(\mathrm{~m}_{\odot}-\mathrm{m}\right)}
$$

where, $\Phi(\alpha)=10^{-0.4 \alpha \beta}$ is the phase function used for the dust grains, $\alpha$ is the phase angle of the observations, and a value of $\beta=0.04 \mathrm{mag} / \mathrm{deg}$ was used for the linear phase coefficient. Additionally, $\mathrm{m}_{\odot}$ is the solar magnitude in the $\mathrm{R}$-band and $\mathrm{m}$ is the R-band magnitude measured for the outburst dust flux. The number of $1.0 \mu \mathrm{m}$ dust grains is found by dividing the total scattering cross section $C_{\text {dust }}$ by the scattering cross section of an assumed spherical individual grain $\left(C_{1.0 \mu m}\right)$. The lower-limit measurement for the amount of material ejected during the outburst is shown in Table 5 for each of the five nights. The amount of material measured for each night is consistent within uncertainties. An average value of $(1.8 \pm$ $0.07) \times 10^{9} \mathrm{~kg}$ was measured as the lower limit to the amount of dust emitted during the outburst.

\subsection{Lower Limit for the Outburst Duration}

To estimate a lower limit for the outburst duration, we present a simple thermal model which includes the sublimation of either pure $\mathrm{CO}$ or $\mathrm{CO}_{2}$ ice. Equations for the thermal modeling are taken from Prialnik et al. 2004, Meech and Svoreň 2004, Sarid et al. 2005, and Sarid 2009. The lower limit is the time required to eject the amount of dust from the surface of SW1 assuming a value for the dust to gas ratio. Our modeling assumptions include: a dust to gas ratio of 4.0 from recent Rosetta spacecraft measurements of Comet 67P (Rotundi et al. 2015), thermal properties of nucleus materials from Huebner et al. 2006, a porosity of $\Psi=0.7$ (Rosetta measurements, Taylor et al. 2015), and an active region comprising $6 \%$ of surface area which is based on our best-fit coma model described in Section 4 . The authors note that there are several other plausible arguments for what drives cometary outbursts, but this idealized calculation of one possibility is shown to help constrain the spin-period outburst-duration relation found during coma morphology modeling.

First, energy balance was used at the nucleus's surface to solve for the temperature, $T$, of the surface area undergoing outburst.

$$
\frac{(1-A) \mathrm{L}_{\odot}}{4 \pi R_{\mathrm{H}}^{2}} \cos z=\epsilon \sigma T^{4}+\sum_{i} \mathcal{F}_{i} P_{i}(T) \sqrt{\frac{\mathrm{m}_{i}}{2 \pi \mathrm{k} T}} H_{i}(T)
$$

In the equation, $\mathrm{L}_{\odot}$ is the solar constant, $A$ is the bolometric albedo of SW1's surface (assuming a geometric albedo of 0.04 and the same phase function as in Section 5.1), $z$ is the local solar zenith angle of the active surface area, $\epsilon$ is the emissivity of the cometary surface (assuming a value of 0.95 found to be standard for most cometary thermal models), $\sigma$ is the Stefan-Boltzmann constant, $P_{i}$ is the saturation vapor pressure of species $i, \mathrm{~m}_{i}$ is the mass of species $i, k$ is the Boltzmann constant, and finally $H_{i}$ is the latent heat of sublimation. Both the saturation vapor pressure and latent heat of sublimation are temperature dependent. Equations from Heubner et al. 2006 were used for both material properties. The factor $\mathcal{F}_{i}$ is the fractional area of the unit surface element covered by the $i$-th sublimating ice species. If pure CO and/or $\mathrm{CO}_{2}$ ice is present and producing the outburst, it will most likely not be at the nucleus' surface as the above 
calculation is assuming. A thermal wave from the surface traveling to subsurface supervolatile ice pockets is probably a better description of the process, but for the simple calculation done here we ignore this fact and proceed as if the supervolatile ices are at the surface. A more thorough analysis is underway and will account for a more detailed analysis of SW1's outburst mechanisms to be presented in the future.

Once the temperature is found, by solving equation (5), the sublimation rate $\left(q_{i}\right)$ for the species is calculated. Equation (6) describes the sublimation per unit surface area.

$$
q_{i}=\left(P_{\mathrm{vap}, i}(T)-P_{i}\right) \sqrt{\frac{m_{i}}{2 \pi \mathrm{k} T}}
$$

where, $P_{\mathrm{vap}, i}(T)$ is the saturation vapor pressure, $P_{i}$ is the vapor pressure of the sublimating gas and assumed to be negligible at the nucleus' surface when compared to the saturation vapor pressure.

Finally, the $6 \%$ active area of SW1's nucleus found from modeling amounts to $7 \times 10^{8} \mathrm{~m}^{2}$, but only a fraction of this surface area consists of $\mathrm{CO}$ and/or $\mathrm{CO}_{2}$ ices. Table 4 shows the total $\mathrm{CO}$ and $\mathrm{CO}_{2}$ production rates for a sampling of possible compositional ratios along with their associated lower-limit outburst duration values. All values of the outburst duration lower limit are on the order of hours, which is plausible given the morphological evolution seen in the observations and the rapid brightening observed during the onset of the outburst. Also, this lower limit is consistent with what one should expect based on tensile strength considerations corresponding to a weakly held nucleus (e.g. Weissman et al. 2004 Comets II chapter).

\section{Conclusions and Discussion}

Investigations into cometary outburst and activity drivers for distant comets is an area of research providing insights into the interior structures of cometary nuclei. Thermophysical modeling of small icy bodies is increasing in complexity allowed by the increasing speeds of modern computer systems. Proper application of these highly detailed models requires knowledge of the nucleus's spin state, allowing proper representation of the nucleus's surface insolation distribution and how it evolves during the orbit. The enigmatic Comet 29P/Schwassmann-Wachmann 1 provides us with an interesting situation in which to test complex thermophysical analysis on a non-spacecraft flyby nucleus due to a large set of both ground- and space-based observations. In this article we have applied a 3-D Monte Carlo coma model to a set of SW1 observations from UT Sept. 2008 shortly after the comet underwent a major outburst. Constraints on the spin state have been found along with measurements of the dust released during the outburst.

The morphological evolution of the outburst coma did not allow constraints to be placed on the spin-pole direction, but constraints were found for the spin period for a set of assumed spin-pole orientations. By sampling a coarse gird in spin-pole orientation space, applying a 3-D Monte Carlo coma model, and thermal modeling we find that a lower limit for the spin period is on the order of days and/or the spin-pole direction during the observations was closely aligned with the sub-Earth direction. The possibly slow rotation rate has an interesting implication, one that is supported by earlier works measuring a spin period on the order of 57-60 days (Trigo-Rodriguez et al. 2010, Miles et al. 2015). Such a long rotation period would only further emphasize SW1 as a unique comet. While we have no evidence for such a slow rotation, it can not be ruled out by our analysis. We find no suggestions of non-principal axis (NPA) rotation, although a complex spin 


\section{Summary of gas-production rates for different assumed material compositions}

\begin{tabular}{|c|c|c|c|c|c|}
\hline $\begin{array}{c}\mathrm{CO} \\
\left(\mathcal{F}_{\mathrm{CO}}\right)^{\mathrm{c}}\end{array}$ & $\begin{array}{c}\mathrm{CO}_{2} \\
\left(\mathcal{F}_{\mathrm{CO}_{2}}\right)^{\mathrm{c}}\end{array}$ & $\begin{array}{c}\mathrm{H}_{2} \mathrm{O} \\
\left(\mathcal{F}_{\mathrm{H}_{2} \mathrm{O}}\right)^{\mathrm{c}}\end{array}$ & $\begin{array}{c}\text { Dust } \\
\left(\mathcal{F}_{\text {Dust }}\right)^{\mathrm{c}}\end{array}$ & $\begin{array}{c}q_{i}^{\mathrm{a}} \\
(\text { molecules } / \mathrm{s})\end{array}$ & $\begin{array}{c}\Delta t^{\mathrm{b}} \\
\text { (hours) }\end{array}$ \\
\hline 0.01 & 0.0 & 0.49 & 0.5 & $1.718 \times 10^{30}$ & 1.56 \\
\hline 0.1 & 0.0 & 0.4 & 0.5 & $1.645 \times 10^{30}$ & 1.63 \\
\hline 0.5 & 0.0 & 0.0 & 0.5 & $1.603 \times 10^{30}$ & 1.68 \\
\hline 1.0 & 0.0 & 0.0 & 0.0 & $1.587 \times 10^{30}$ & 1.69 \\
\hline 0.0 & 0.01 & 0.49 & 0.5 & $4.143 \times 10^{29}$ & 4.13 \\
\hline 0.0 & 0.1 & 0.4 & 0.5 & $4.457 \times 10^{29}$ & 3.84 \\
\hline 0.0 & 0.5 & 0.0 & 0.5 & $4.598 \times 10^{29}$ & 3.72 \\
\hline 0.0 & 1.0 & 0.0 & 0.0 & $4.645 \times 10^{29}$ & 3.68 \\
\hline
\end{tabular}

${ }^{a}$ Gas production rate assuming the fraction of active area is composed of the supervolatile species.

b Minimum time required to eject the measured dust produced during the outburst assuming a dust to gas ratio of four from recent Rosetta mission results (Taylor et al. 2015).

${ }^{\mathrm{c}}$ Fraction of surface area element composed of the specified ice used in Equation 5. outburst? To test this mechanism we applied a simple thermal model to the $\sim 6 \%$ active surface region found from 3 -D Monte Carlo coma modeling which incorporated either $\mathrm{CO}$ or $\mathrm{CO}_{2}$ ice sublimation. The measured lower limit $\left(1.8 \times 10^{9}\right.$ $\mathrm{kg}$ ) of dust ejected during the outburst could be released through sublimation of either ice with a timescale on the order 
ID: 0470; PI: B. Mueller), which is operated by the Association of Universities for Research in Astronomy (AURA) under cooperative agreement with the National Science Foundation. We acknowledge funding support from NASA's Outer Planets Research program (grant NNX12AK50G). We would also like to thank the article's reviewer Matthew Knight and an anonymous reviewer for their comments and suggestions during the preparation of this article.

Science Foundation, and the U.S. Department of Energy Office of Science. The SDSS-III web site is http://www.sdss3.org/.

SDSS-III is managed by the Astrophysical Research Consortium for the Participating Institutions of the SDSS-III Collaboration including the University of Arizona, the Brazilian Participation Group, Brookhaven National Laboratory, Carnegie Mellon University, University of Florida, the French Participation Group, the German Participation Group, coma modeling and tensile strength considerations.

While we were unable to place constraints on the spin-pole direction with this analysis, we were able to constrain SW1's nucleus to a slow rotation state. Current and future projects by our group are working to place constraints on the spin-pole directions and/or to confirm the slow rotation rate. If SW1's nucleus is indeed found to be in a slow spin state, the spin-pole orientation may be of less importance with regards to future thermophysical modeling.

\section{To summarize:}

- Five consecutive nights of observations of SW1 $\sim 4$ days after a major outburst were analyzed.

- An expanding shell of material was measured to have a projected velocity of $0.11 \pm 0.02 \mathrm{~km} / \mathrm{s}$ due to the outburst.

- Linear features on the northern side of the outburst coma were found to be contained in the expanding shell of material and originate from the same event producing the shell.

- 3-D Monte Carlo coma modeling of the morphological evolution of the outburst coma suggest either a slow (on the order of days) rotation period or a spin-pole directions directed towards Earth during observations.

- Comparing radial surface-brightness profiles of the observations to synthetic comet images generated using the coma models provide an upper limit for the outburst duration of $\sim 1.5$ days.

- Using a simple thermal model to describe the outburst event and the total amount of dust emitted during the outburst, a lower limit for the outburst duration was calculated to be on the order of hours.

- Photometry of the outburst resulted in an estimate of $(1.8 \pm 0.07) \times 10^{9} \mathrm{~kg}$ as a lower limit to the total amount of dust ejected during the outburst.

\section{Acknowledgements}

Funding for SDSS-III has been provided by the Alfred P. Sloan Foundation, the Participating Institutions, the National Harvard University, the Instituto de Astrofisica de Canarias, the Michigan State/Notre Dame/JINA Participation Group, Johns Hopkins University, Lawrence Berkeley National Laboratory, Max Planck Institute for Astrophysics, Max Planck 
Institute for Extraterrestrial Physics, New Mexico State University, New York University, Ohio State University, Pennsylvania State University, University of Portsmouth, Princeton University, the Spanish Participation Group, University of Tokyo, University of Utah, Vanderbilt University, University of Virginia, University of Washington, and Yale University.

\section{References}

A'Hearn, M. F., et al.: 1984, Comet Bowell 1980b, The Astronomical Journal 89, 579.

Crifo, J. F., et al.: 1999, The Dependence of the Circumnuclear Coma Structure on the properties of the Nucleus, III. First Modeling of a CO-Dominated Coma, with Applications to Comets 46P/Wirtanen and 29P/SchwassmannWachmann 1, Icarus 138, 85-106.

Farnham, T. L.: 2009, Coma morphology of Jupiter-family comets, Planetary and Space Science 57, 1192-1217.

Fernández, Y. R., et al.: 2012, Properties of the Nucleus, Dust Coma, and Gas Coma of Comet 29P/SchwassmannWachmann 1 As Observed By WISE/NEOWISE, AAS/Division for Planetary Sciences Meeting Abstracts, 44 314.19 .

Fernández, Y. R., et al.: 2013, Thermal properties, sizes, and size distribution of Jupiter-family cometary nuclei, Icarus, 226 1138-1170.

Festou et al., 2001, The Activity of Comet 29P/Schwassmann-Wachmann 1 Monitored through Its CO J=2->1 Radio Line, Icarus 150, 140-150.

Gronkowski, P., 2014, The outburst of the comet 29P/Schwassmann-Wachmann 1: A new approach to the old problem, Astron. Nachr. AN 2, No. 335, 124-134.

Gronkowski, P. and Wesołowski M.: 2015, A model of cometary outbursts: a new simple approach to the classical question, Monthly Notices of the Royal Astronomical Society 451, 3068-3077.

Gunnarsson et al., 2001, An Extended CO Source around Comet 29P/Schwassmann-Wachmann 1, Icarus 157, 309-322.

Huebner, W. F., et al.: 2006, Heat and Gas Diffusion in Comet Nuclei, ISSI Scientific Report, SR-004.

Hosek, M. W., et al.: 2013, The Astrophysical Journal 145, 122.

Ivanova, O. V., et al.: 2011, Icarus 211, 559.

Jewitt, D. C., 2009, The Active Centaurs, The Astronomical Journal 137, 4296-4312.

Kossacki, K. J., and Szutowicz, S., 2013, Activity of Comet 29P/Schwassmann-Wachmann 1, Icarus 225, 111-121.

McCarthy, D. W., et al.: 2007, Comet Hale-Bopp in outburst: Imaging the dynamics of icy particles with HST/NICOMS, Icarus 189, 184-195. 
Meech, K. J., et al.: 1993, Nucleus Properties of P/Schwassmann-Wachmann 1, The Astronomical Journal 106, $1222-1236$.

Meech, K. J., and Svoreň, J.: 2004, Using Cometary Activity to Trace the Physical and Chemical Evolution of Cometary Nuclei, Comets II, 317-335.

Miles, R., et al.: 2016, Anatomy of outburst and quiescent activity of Comet 29P/Schwassmann-Wachmann, Icarus 272, 327-355.

Mueller, B. E.A., et al.: 2013, Analysis of the sunward continuum features of Comet 103P/Hartley 2 from groundbased images, Icarus 222, 799-807.

Paganini, L., et al., 2013, Ground-Based Infrared Detections of CO in the Centaur-Comet 29P/SchwassmannWachmann 1 at 6.26 AU from the Sun, The Astrophysical Journal 766, 100.

Prialnik, D., et al.: 2004, Modeling the Structure and Activity of Comet Nuclei, Comets II, 359-387.

Samarasinha, N. H.: 2000, The Coma Morphology Due to an Extended Active Region and the Implications for the Spin Sate of Comet Hale-Bopp, The Astrophysical Journal 529, L107-L110.

Samarasinha, N. H., et al.: 2004, Rotation of Cometary Nuclei, Comets II, 281-299.

Samarasinha, N. H., et al.: 2011, Rotation of Comet 103P/Hartley 2 from Structures in the Coma, The Astrophysical Journal Letters 734:L3 (6pp).

Samarasinha, N. H. and Larson, S. M.: 2014, Image enhancement techniques for quantitative investigations of morphological features in cometary comae: A comparative study, Icarus 239, 168-185.

Sarid, G., et al.: 2005, Thermal Evolution and Activity of Comet 9P/Tempel 1 and Simulation of a Deep Impact, Publications of the Astronomical Society of the Pacific 117, 796-809.

Sarid, G.: 2009, Thermal and Structural Evolution of Small Bodies in the Solar System, PhD Thesis, Tel Aviv University.

Schambeau, C. A., et al.: 2015, A new analysis of Spitzer observations of Comet 29P/Schwassmann-Wachmann 1, Icarus 260, 60-72.

Schleicher, D. G., and Woodney, L. M.: 2003, Analysis of dust coma morphology of Comet Hyakutake (1996 B2) near perigee: outburst behavior, jet motion, source region locations, and nucleus pole orientation, Icarus 162, $190-213$.

Sekanina, Z. and Larson, S. M.: 1984, Coma Morphology and Dust-Emission Pattern of Periodic Comet Halley. II. Nucleus Spin Vector and Modeling of Major Dust Features in 1910, The Astronomical Journal 89, 1408-1425.

Trigo-Rodriguez, J. M., et al., 2010, Outburst activity in comets - II. A multiband photometric monitoring of comet 29P/Schwassmann-Wachmann 1, Mon. Not. R. Astron. Soc., 409, 1682-1690.

Weissman, P. R., et al.: 2004, Structure and Density of Cometary Nuclei, Comets II, 337-357. 